Pacific Journal of Mathematics

NORMS OF POWERS OF ABSOLUTELY CONVERGENT 


\title{
NORMS OF POWERS OF ABSOLUTELY CONVERGENT FOURIER SERIES: AN EXAMPLE
}

\author{
Charles H. Heiberg
}

Let $f$ be defined on $T^{2}$ and have an absolutely convergent Fourier series

$$
f\left(e^{i \sigma_{1}}, e^{i \sigma_{2}}\right)=\sum f_{m} e^{i m_{1} \sigma_{1}} e^{i m_{2} \sigma_{2}}
$$

Set $\|f\|=\Sigma\left|f_{m}\right|$ and $\|f\|_{2}^{2}=\Sigma\left|f_{m}\right|^{2}$. In this paper the asymptotic behavior of $\left\|f^{k}\right\|$, as $k \rightarrow \infty$, is studied.

THEOREM 1. Let $f$ be a continuous function on $T^{2}$ such that

$$
|f(z)|<1 \text { for } z \neq(1,1), \quad\left|z_{1}\right|=\left|z_{2}\right|=1
$$

and such that for all $\sigma$ in some $R^{2}$ neighborhood of $(0,0)$

$$
f\left(e^{i \sigma}\right)=\exp (i \lambda(\sigma)-\psi(\sigma))
$$

where $\lambda$ is a real-valued linear function defined on $R^{2}$ and $\psi$ is a continuous, complex-valued valued function defined on $R^{2}$ and satisfying certain smoothness conditions to be defined near the end of $\$ 2$ below. Then
(i) $\left\|f^{k}\right\|_{2}^{2} \leqq a k^{-1 / p} \log k$,
(ii) $\sup _{m}\left|\left(f^{k}\right)_{m}\right| \leqq a k^{-1 / p} \log k$,
(iii) $b k^{-1 / p} \log k \leqq \sup _{m} \operatorname{Re}\left(f^{k}\right)_{m}$,
(iv) $b \log k \leqq \Sigma_{m}\left|\operatorname{Re}\left(f^{k}\right)_{m}\right|$.

THEOREM 2. There exists a polynomial $f$ in two complex variables satisfying $f(1,1)=1$ and

$$
|f(z)|<1 \text { for } z \neq(1,1), \quad\left|z_{1}\right|=\left|z_{2}\right|=1,
$$

such that

(i) $\left\|f^{k}\right\| \leqq b \log k$,

(ii) $a \log k \leqq\left\|f^{k}\right\|$,

(iii) $a \log k \leqq\left\||f|^{k}\right\|$,

(iv) $\left\|f^{k}\right\|_{2}^{2} \leqq b \sup _{m}\left|\left(f^{k}\right)_{m}\right|$.

In 1970 B. M. Schreiber published smoothness conditions which, for functions defined on $T^{q}$, having absolutely convergent Fourier series and 
satisfying a condition that reduces to (1) in the case $q=2$, imply that $\left\|f^{k}\right\|$ remains bounded as $k$ tends to infinity.

1. Introduction. It is a consequence of Theorem 2 that certain well-known results concerning functions $f(z)$ analytic on the closed unit disc do not extend to even the case of functions of two complex variables. Namely, letting

$$
\begin{aligned}
& f^{k}(z)=\sum a_{m, k} z^{m}, \\
& \left\|f^{k}\right\|=\sum\left|a_{m, k}\right|, \quad\left\|f^{k}\right\|_{2}^{2}=\sum\left|a_{m, k}\right|^{2},
\end{aligned}
$$

it is known that if $f(1)=1$ and $|f(z)|<1$ for $z \neq 1,|z|=1$, then

(i) $\quad\left\||f|^{k}\right\|=O(1)$, as $k \rightarrow \infty$, (see [5]),

(ii) $a k^{b} \leqq\left\|f^{k}\right\| \leqq c k^{b}$, if $\left\|f^{k}\right\| \rightarrow \infty$, as $k \rightarrow \infty$, (see [5], [3]),

(iii) $\left\|f^{k}\right\|_{2}^{2} / \sup _{m}\left|a_{m, k}\right| \rightarrow \infty$, if $\left\|f^{k}\right\| \rightarrow \infty$ as $k \rightarrow \infty$, (see [1], [2]).

None of these results extend to functions of two complex variables.

The results of Bajsanski [1] and Clunie and Vermes [2] were rediscovered and appear in [7], [9], [10]. For other results concerning norms of powers of absolutely convergent Fourier series of several variables see [4], [6], [8].

2. Notation and remarks. Let $T, Z, R$ and $C$ denote the unit circle, integers, real numbers and complex numbers respectively. For any set $S$ let $S^{2}$ denote the cartesian product of the set with itself. The letters $\sigma$ and $z$ denote points of $R^{2}$ and $C^{2}$ respectively, $\sigma_{1}, \quad \sigma_{2}$ and $z_{1}, z_{2}$ their respective components and $e^{z}$ the point $\left(e^{z_{1}}, e^{z_{2}}\right)$. For $p$ in $Z^{2}, \sigma^{p}$ denotes $\sigma_{1}^{p_{1}} \sigma_{2}^{p_{2}}$. For $p=2,3, \cdots, \sigma^{p}$ denotes $\sigma_{1}^{p} \sigma_{2}^{p}$. The scalar product of two points $\sigma$ and $\lambda$ of $R^{2}$ will be denoted by $\lambda \cdot \sigma$. Since $R^{2}$ is its own dual the same notation is used for a point $\lambda$ of $R^{2}$ and the corresponding linear map $\lambda: \sigma \rightarrow \lambda \cdot \sigma$. For any subset $S$ of $R^{2}$ and point $r$ of $R$ let $r S$ denote the set of points $r s$ such that $s$ belongs to $S$.

Denote $[-\pi, \pi]^{2}$ by $\Pi$ and the complement in $\Pi$ of any subset $S$ of $\Pi$ by $C S$.

The letters $a, b, c, d$ will denote absolute positive constants. The use of one such letter in two inequalities does not mean that the letter represents the same absolute constant in both inequalities. However, the use of such a letter in an inequality involving the indices $k$ and $m$ means that the constant represented by the letter is independent of $k$ and $m$. The phrases "for $k$ sufficiently large" and "for all $m$ " will be omitted finitely many times from this paper. 
For positive, even integers $p$ and $q$ let $\Phi_{p, q}$ denote the class of continuous complex-valued functions $\psi$ defined on $R^{2}$ for which

$$
|\phi(\sigma)-\psi(\sigma)| / \phi(\sigma)=O\left(\log ^{-2}|\sigma|\right), \text { as }|\sigma| \rightarrow 0,
$$

for some polynomial $\phi$ of the form

$$
\alpha\left(\sigma^{p}+\sigma_{1}^{q}+\sigma_{2}^{q}\right), \quad \alpha>0 .
$$

Then a function $\psi$ satisfies the smoothness conditions mentioned in Theorem 1 if and only if there exist positive even integers $p$ and $q$ such that $\psi$ belongs to $\Phi_{p, q}$ and such that $2 p<q$.

Finally, note that for Theorem 1 it is not assumed that the Fourier series of $f$ is absolutely convergent.

\section{Proofs of theorems.}

Lemma 1. Fix $p>1$. Let $F(t)$ denote the integral of $\exp \left(-b x^{p}\right)$ over $(t, \infty)$. Then

(i) $\int_{0}^{\beta} \int_{0}^{a} \exp \left(-b s^{p} t^{p}\right) d s d t \sim F(0) \log \beta$, as $\beta \rightarrow \infty$. Consequently, if $p$ is a positive even integer, then

(ii) $\int_{-\beta a}^{\beta a} \int_{-\beta a}^{\beta a} \exp \left(-b s^{p} t^{p}\right) d s d t \sim 8 F(0) \log \beta$, as $\beta \rightarrow \infty$.

Proof. Let $\epsilon>0$ be given and let $I(\beta)$ denote the double integral in (i). By substituting $x / t$ for $s$ in $I$ it can be seen that for $\beta>1$

$$
F(0) \log \beta-I(\beta)=\int_{0}^{1}(F(a t)-F(0)) / t d t+\int_{1}^{\beta} F(a t) / t d t .
$$

Choose $T>1$ so that $F(a t)<\epsilon F(0)$ for all $t$ in $(T, \infty)$. The function $F(t) / t$ is bounded on $(1, T)$ so it follows from the last equality that

$$
|F(0) \log \beta-I(\beta)| \leqq c+\epsilon F(0) \log \beta .
$$

Since $\epsilon$ was arbitrary

$$
\left|1-I(\beta)(F(0) \log \beta)^{-1}\right| \leqq c(F(0) \log \beta)^{-1}
$$

for $\beta>1$, which proves (i).

To prove (ii) substitute $x \beta$ for $s$ and $y \beta^{-1}$ for $t$ in the integral in (ii), use that the integrand is an even function of each variable to obtain an integral over $(0, a) \times\left(0, \beta^{2} a\right)$ only and apply (i). 
Proof of Theorem 1(i). From (2), (3) and the definition of the function $\phi$ it follows that

$$
\left|f\left(e^{i \sigma}\right)\right| \leqq \exp (-c \phi(\sigma))
$$

for all $\sigma$ in some neighborhood of $(0,0)$. Since $|f|$ satisfies (1) and is continuous, the inequality (4) holds on all of $T^{2}$. Thus,

$$
\left\|f^{k}\right\|_{2}^{2} \leqq \int_{\Pi} \exp \left(-k c \sigma^{p}\right) d \sigma
$$

Substituting $k^{-1 / 2 p} \tau$ for $\sigma$ in this last integral and applying Lemma 1(ii) completes the proof of (i).

Proof of Theorem 1(ii). If $f$ satisfies the hypothesis of (i), then $|f|^{\frac{1}{2}}$ does also, so

$$
\left\||f|^{k / 2}\right\|_{2}^{2} \leqq a k^{-1 / p} \log k
$$

This and the inequality

$$
\sup _{m}\left|\left(f^{k}\right)_{m}\right| \leqq\left\||f|^{k / 2}\right\|_{2}^{2}
$$

prove (ii).

Define on $\Pi$ an auxiliary function $A$ which is used in estimating the Fourier coefficients of $f^{k}$. Let

$$
A=\exp (i \lambda-\phi)
$$

and note that

$$
|A| \leqq \exp (-\phi)
$$

since $\lambda$ is real-valued.

Lemma 2. For $i=1,2$ there exists a collection of sets $\left\{E_{i}(k, m)\right\}$ indexed by $k$ in $Z, m$ in $Z^{2}$ such that

$$
\sup _{m}\left|\left(f^{k}\right)_{m}-\left(\sum_{i} \chi_{E_{i}} A^{k}\right)_{m}\right| \leqq c k^{-1 / p}
$$

Proof. For each $k$ in $Z, m$ in $Z^{2}$ let 
(7)

$$
\begin{aligned}
& \text { (i) } \nu(k, m)=k \lambda-m \text {, (ii) } \rho^{2}(k, m)=\left|\nu_{1} \nu_{2}\right| k^{-1 / p} \text {, } \\
& S=\left\{\sigma \in \Pi:\left|\sigma_{\imath}\right|<\rho /|\nu|_{\imath}\right\}, \\
& D=\left\{\sigma \in \Pi:\left|\sigma_{\imath}\right|>\rho /|\nu|_{i}\right\} \text {, } \\
& E_{\imath}=\left\{\sigma \in \Pi:\left|\sigma_{\imath}\right|>\rho /|\nu|_{\imath},\left|\sigma_{3-i}\right|<\rho /|\nu|_{3-i}\right\},
\end{aligned}
$$

$i=1,2$. Then

$$
\left(f^{k}\right)_{m}-\left(\sum \chi_{E_{t}} A^{k}\right)_{m}=\sum_{j=1}^{3} I_{j}
$$

where, for $j=1,2,3, I$ is the $m$ th Fourier coefficient of $f^{k}-A^{k}, \chi_{s} A^{k}$, $\chi_{D} A^{k}$ respectively.

Since by (6) $|A| \leqq 1$ the integral $I_{2}$ can be estimated by the area of $S$ and hence

$$
\left|I_{2}\right| \leqq 4 \rho^{2} /\left|\nu_{1} \nu_{2}\right|=4 k^{-1 / p}
$$

the equality resulting from (7)(ii).

To estimate $I_{1}$ let

$$
W_{k}=\{\sigma \in \Pi: k \phi(\sigma) \leqq(1+1 / c) \log k\},
$$

where $c$ is given in (4), and write

$$
\left|I_{1}\right| \leqq\left(\int_{W_{k}}+\int_{C W_{k}}\right)\left|f^{k}-A^{k}\right|
$$

To estimate $\int_{C W_{k}}$, the second integral in (11), estimate the sum of the supremums over $C W_{k}$ of $|f|^{k}$ and $|A|^{k}$. Since on $C W_{k}$

$$
\begin{aligned}
& \exp (-c k \phi) \leqq \exp (-(c+1) \log k) \\
& \exp (-k \phi) \leqq \exp (-(1+1 / c) \log k)
\end{aligned}
$$

it follows from (4) and (6) that the supremums to be estimated are $o\left(k^{-1}\right)$, as $k \rightarrow \infty$. Since the measure of $C W_{k}$ is bounded by $4 \pi^{2}$ it follows that

$$
\int_{C w_{k}}=o\left(k^{-1}\right), \text { as } k \rightarrow \infty \text {. }
$$

To estimate $\int_{W_{k}}$, as given in (11), note that (2) holds on $W_{k}$, for $k$ sufficiently large, so that $f=\exp (\phi-\psi) \cdot A$ on $W_{k}$ and so that 
(13)

$$
\int_{W_{k}}<\sup _{W_{k}}|\exp k(\phi-\psi)-1| \int_{\Pi} \exp \left(-\alpha k \sigma^{p}\right) d \sigma .
$$

By (3),

$$
\sup _{w_{k}}|\phi-\psi| \leqq a \sup _{w_{k}}\left(\phi \log ^{-2}|\sigma|\right)
$$

Using that $|\sigma| \leqq\left|\sigma_{1}\right|+\left|\sigma_{2}\right|$ and the definition of $W_{k}$ yields for some positive number $\epsilon$

$$
\sup _{w_{k}}\left(\log ^{-2}|\sigma|\right) \leqq \log ^{-2}\left(2 k^{-\epsilon}\right)
$$

and

$$
\sup _{W_{k}} \phi \leqq k^{-1} \log k
$$

The last three estimates involving supremums imply that

$$
\sup _{w_{k}}|\phi-\psi|=O\left((k \log k)^{-1}\right), \text { as } k \rightarrow \infty,
$$

and hence that

$$
\sup _{w_{k}}|\exp k(\phi-\psi)-1|=O\left((\log k)^{-1}\right) \text {, as } k \rightarrow \infty \text {, }
$$

since

$$
\exp k(\phi-\psi)=\sum_{\mu=0}^{\infty}(k|\phi-\psi|)^{\mu} / \mu !
$$

The second integral in (13), being of the same type as the integral in (5), is $O\left(k^{-1 / p} \log k\right)$, as $k \rightarrow \infty$. This and (11)-(14) prove that

$$
\left|I_{1}\right|=O\left(k^{-1 / p}\right), \text { as } k \rightarrow \infty \text {. }
$$

From the definition of $A$ it follows that

$$
\left|I_{3}\right| \leqq 4 \int_{D} \exp \left(-\alpha k \sigma^{p}\right) d \sigma
$$


By substituting $\rho \tau /|\nu|$ for $\sigma$ and using (7)(ii) and that $\exp \left(-\alpha \tau^{p}\right)$ is integrable on $(1, \infty)^{2}$ conclude that $\left|I_{3}\right| \leqq 4 k^{-1 / p}$. This, (15), (8) and (9) prove Lemma 2.

Since $2 p<q$ there exists $\epsilon$ in $(0,1)$ so that

$$
2 p<q \epsilon
$$

For each $k$ in $Z$ and positive constant $b$ let $M_{b}(k)$ denote the set of points $m$ of $Z^{2}$ such that $\nu(m)$ and $\rho(m)$, defined by (7), satisfy

$$
\text { (i) } k^{1 / q \epsilon} \leqq \min \left|\nu_{1}\right|, i=1,2 \text { and (ii) } \rho^{2}<b
$$

LEMMA 3. For all $k$ sufficiently large

$$
\inf \left(\chi_{E_{t}} A^{k}\right)_{m}|\log \rho|^{-1} \geqq a k^{-1 / p}, \quad i=1,2,
$$

where the infimum is over all $m$ in $M_{b}(k)$.

Proof. The proof is given for $i=1$, the proof for $i=2$ being similar. Since $p$ and $q$ are even integers, $\phi$ is an even function of $\sigma_{1}$ and of $\sigma_{2}$. It follows that $\chi_{E_{1}} A^{k}$ is also and that

$$
\pi^{2}\left(\chi_{E_{t}} \cdot A^{k}\right)_{m}=\int_{0}^{\rho /\left|\nu_{2}\right|} \int_{0}^{\pi} I(\sigma) d \sigma_{1} d \sigma_{2}
$$

where $I(\sigma)=\cos \left(\nu_{1} \sigma_{1}\right) \cos \left(\nu_{2} \sigma_{2}\right) \exp (-k \phi)$.

Since for some $\gamma$ in $(0,1),|I(\sigma)|^{\frac{1}{2}} \leqq \gamma^{k}$ for $\sigma_{1} \geqq \pi$ and since $|I(\sigma)|^{\frac{1}{2}}$ is integrable on $R^{2}$ it follows from (18) that

$$
\left|\int_{0}^{\rho /\left|v_{2}\right|} \int_{\pi}^{\infty} I(\sigma) d \sigma_{1} d \sigma_{2}\right|=O\left(\gamma^{k}\right), \text { as } k \rightarrow \infty .
$$

Using the points $\pi / 8\left|\nu_{1}\right|, 3 \pi / 8\left|\nu_{1}\right|, \pi / 2\left|\nu_{1}\right|$ partition $\left(0,3 \pi / 2\left|\nu_{1}\right|\right)$ into four subintervals $I_{j}, 0 \leqq j \leqq 3$, and write

$$
\int_{0}^{\rho /\left|\nu_{2}\right|} \int_{0}^{\infty} I(\sigma) d \sigma_{1} d \sigma_{2}=\sum_{j=0}^{4} J_{J}
$$

where $J_{j}$ is the integral of $I$ over the rectangle $R_{j}$ defined to be $I_{1} \times\left(0, \rho\left|\nu_{2}\right|^{-1}\right)$, for $0 \leqq j \leqq 3$, and where $J_{4}$ is defined by (20).

Requiring that $b \leqq 1$ it follows from (17) and the definition of $R_{0}$ that for $\sigma$ in $R_{0}$

$$
\max \sigma_{i} \leqq k^{-1 / q \epsilon} .
$$


Since $\epsilon$ is in $(0,1)$ it follows that for all $k$ sufficiently large $\exp \left(-k \alpha\left(\sigma_{1}^{q}+\sigma_{2}^{q}\right)\right) \geqq \frac{1}{2}$, for all $\sigma$ in $R_{0}$. Also $\cos \left(\nu_{1} \sigma_{1}\right)$ and $\cos \left(\nu_{2} \sigma_{2}\right)$ exceed $\frac{1}{2}$ for all $\sigma$ in $R_{0}$. Thus,

$$
8 J_{0} \geqq \int_{R_{0}} \exp \left(-k \alpha \sigma^{p}\right) d \sigma .
$$

By substituting $x\left|\nu_{1}\right|^{-1}$ for $\sigma_{1}$ and then $y\left|\nu_{2}\right|^{-1} \rho^{2}$ for $\sigma_{2}$ in this last double integral, by using 7(ii) and then by applying Lemma 1(i) it follows that there exist constants $a$ and $b$ such that

$$
J_{0} \geqq a k^{-1 / p}|\log \rho|
$$

for all $m$ in $M_{b}(k)$.

Since $\rho \leqq 1$ it follows for each fixed $\sigma_{2}$ in $\left(0, \rho /\left|\nu_{2}\right|\right)$ that $\cos \left(\nu_{2} \sigma_{2}\right) \exp (-k \phi(\sigma))$ is a monotone decreasing function of $\sigma_{1}$ on $(0, \infty)$ and hence that $J_{4} \geqq 0$.

Since $I$ is positive on $R_{2}, J_{2} \geqq 0$.

To estimate $J_{1}+J_{3}$ substitute for $\sigma_{1}$ in $J_{3}$ in such a way that the limits of the integrals $J_{1}$ and $J_{3}$ become identical, combine integrals and factor the integrand to obtain that

$$
J_{1}+J_{3}=\int_{R_{1}} \cos \left(\nu_{2} \sigma_{2}\right) \exp \left(-k \phi\left(4 \sigma_{1}, \sigma_{2}\right)\right) H(\sigma) d \sigma
$$

$H$ being continuous and defined by the equality.

We note that the last integrand is bounded absolutely (independent of $k$ and $m$ ) and shall show that there exists a rectangle $R_{4}$ such that the last integrand is positive on $R_{1} \backslash R_{4}$ and such that the area of $R_{4}$ is $O\left(k^{-1 / p}\right)$, as $k \rightarrow \infty$.

It will follow that $J_{1}+J_{3} \geqq-d k^{-1 / p}$ which with (18)-(21) and the fact that $J_{2}$ and $J_{4}$ are positive prove the lemma.

Let $R_{4}=I_{1} \times\left(0,3\left|\nu_{1}\right|(k \alpha)^{-1 / p}\right)$.

On $I_{1}, \cos \left(\nu_{1} \sigma_{1}\right) \geqq 1 / 3$ so

$$
H(\sigma) \geqq\left(\exp \left(k \phi\left(4 \sigma_{1}, \sigma_{2}\right)-k \phi(\sigma)\right)\right) / 3-4 .
$$

But the argument of the exponential is bounded from below by $\alpha k\left(4^{p}-\right.$ 1) $\sigma^{p}$, which is never less than 12 on $R_{1} \backslash R_{4}$, since $p$ is an even integer. It follows that $H$ and hence the integrand in (22) are positive on $R_{1} \backslash R_{4}$. This and the fact that the area of $R_{4}$ is $O\left(k^{-1 / p}\right)$, as $k \rightarrow \infty$, prove the lemma. 


$$
\operatorname{Re}\left(f^{k}\right)_{m} \geqq(a|\log \rho|-c) k^{-1 / p},
$$

for all $m$ in $M_{b}(k)$. For $\rho_{0}$ sufficiently small

$$
\operatorname{Re}\left(f^{k}\right)_{m} \geqq d k^{-1 / p}|\log \rho|
$$

for all $m$ in $M_{\rho_{0}}(k)$.

Choose for each positive integer $k$, points $m(k)$ in $Z^{2}$ such that

$$
k^{1 / q \epsilon} \leqq\left|\nu_{l}\left(k, m_{\imath}(k)\right)\right| \leqq 2 k^{1 / q \epsilon}, \quad i=1,2 .
$$

By 7(ii) and this choice

$$
\rho^{2}(k, m(k)) \leqq 4 k^{2 / q \epsilon-1 / p}
$$

which by (16) implies that

$$
\rho^{2}(k, m(k))<\rho_{0}
$$

for all $k$ sufficiently large. This and (24) imply that $m(k)$ belongs to $M_{\rho 0}(k)$ and hence that

$$
\operatorname{Re}\left(f^{k}\right)_{m} \geqq d k^{-1 / p}|\log \rho(k, m(k))| \text {. }
$$

This, (25) and (16) prove (iii).

Proof of Theorem 1(iv). Since $m$ belonging to $M_{\rho_{0}}(k)$ implies that $\rho^{2}<\rho_{0}$ and since $\rho_{0}<1$, inequality (23) implies that

$$
\operatorname{Re}\left(f^{k}\right)_{m} \geqq c k^{-1 / p}
$$

for all $m$ in $M_{\rho_{0}}(k)$. So to prove (iv) it suffices to show that the cardinality of the set $M_{\rho 0}(k)$ exceeds

$$
a k^{1 / p}(1 / p-2 / q \epsilon) \log k
$$

where by (16) the constant in parentheses is positive.

For this proof only, extend the definitions of $\nu$ and $\rho$ (see (7)) to all $k$ in $Z, \sigma$ in $R^{2}$ and define $S_{b}(k)$ to be the set of points $\sigma$ of $R^{2}$ such that $\nu$ and $\rho$ satisfy (17). It suffices to show that if for some $m$ in $Z^{2}$ the set $S_{4 \rho_{0}}(k)$ intersects the square $\left(m_{1}, m_{1}+1\right) \times\left(m_{2}, m_{2}+1\right)$, then $m$ belongs to $M_{\rho_{0}}(k)$, since then the cardinality of $M_{\rho_{0}}(k)$ is no less than the area of $S_{4 \rho_{0}}(k)$ which is no less than the expression in (26). 
Let $\sigma$ be a point of intersection. Since $\sigma$ belongs to $S_{\frac{1}{4 \rho_{0}}}(k)$ then

$$
\nu_{\imath}\left(k, \sigma_{\imath}\right) \geqq k^{1 / q \epsilon}
$$

and since $\sigma$ belongs to $\left(m_{1}, m_{1}+1\right) \times\left(m_{2}, m_{2}+1\right)$ then

$$
0<\nu_{\imath}\left(k, m_{i}\right)-\nu_{\imath}\left(k, \sigma_{\imath}\right)<1, \quad i=1,2 .
$$

It follows that $m$ satisfies (17)(i) and that

$$
\nu_{i}\left(k, m_{\imath}\right) \leqq 2 \nu_{i}\left(k, \sigma_{\imath}\right) \text { for } k \geqq 2^{q \epsilon} .
$$

Using (7) to express $\rho$ as a product of the $\nu_{\imath}$ obtain that

$$
\rho^{2}(k, m) \leqq 4 \rho^{2}(k, \sigma) .
$$

This and the fact that $\sigma$ belongs to $S_{\frac{1}{4 \rho_{0}}}(k)$ imply that $m$ satisfies (17)(ii).

To prove Theorem 2 a polynomial $f$ is defined and Lemmas 4 and 5 are used to show that $f$ and consequently $|f|$ satisfy the conditions given in the hypothesis of Theorem 1 . It follows by Theorem 1 that the inequalities given in (ii) and (iv) and hence (iii) of Theorem 2 hold for both $f$ and $|f|$. Lemmas 2 and 6 are used in a rather difficult proof that $f$ satisfies the inequality given in Theorem 2(i).

The proofs of the next two lemmas follow directly from Lemmas 1 and 2(i) in Heiberg [6].

LEMMA 4. Let $n$ be a positive integer and

$$
g(w)=w^{n}+(-1)^{n-1}\left(\frac{w-1}{2}\right)^{2 n}
$$

for all complex $w$. Then

(i) $|g(w)|<1$ for $|w|=1, w \neq 1$,

(ii) $g\left(e^{i s}\right)=\exp \left(n s i-\sum_{j=2 n}^{\infty} d_{j} s^{j}\right), d_{2 n}>0$, for all $s$ in some neighborhood of 0 .

LEMMA 5. Let $p$ be a positive integer and $\sum c_{j} \sigma^{\prime}$ converge absolutely for all $\sigma$ in some neighborhood of $(0,0)$, where the sum is over all $j$ in $Z^{2}$ such that $j_{1}+j_{2}>2 p ; j_{1}, j_{2} \geqq p$. Then

$$
\left|\Sigma c_{j} \sigma^{j}\right|=O\left(|\sigma|\left|\sigma^{2 p}\right|\right) \text {, as }|\sigma| \rightarrow 0
$$


Lemma 6. For all $k$ sufficiently large

$$
\sup _{m}\left|\left(\chi_{E_{i}} \cdot A^{k}\right)_{m}\right|(|\log \rho|+1)^{-1} \leqq a k^{-1 / p}, \quad i=1,2
$$

Proof. The last inequality is proved for $i=1$, the proof for $i=2$ being similar.

By (18) it suffices to prove that

$$
\int_{0}^{\rho / \nu_{2} \mid} \int_{0}^{\pi} I(\sigma) d \sigma_{1} d \sigma_{2} \leqq a k^{-1 / p}(|\log \rho|+1)
$$

for all $m$ in $Z^{2}$.

If $3 \pi / 2\left|\nu_{1}\right|<\pi$, then for each fixed $\sigma_{2}$ the integral, $\int_{h}^{\pi} I(\sigma) d \sigma$, is of opposite signs for $h=3 \pi / 2\left|\nu_{1}\right|$ and $h=\pi / 2\left|\nu_{1}\right|$ since for each fixed $\sigma_{2}$, $\phi(\sigma)$ is a monotone function of $\sigma_{1}$. It follows that

$$
\left|\int_{\pi / 2\left|\nu_{1}\right|}^{\pi} I(\sigma) d \sigma_{1}\right| \leqq\left|\int_{\pi / 2\left|\nu_{1}\right|}^{3 \pi / 2\left|\nu_{1}\right|} I(\sigma) d \sigma_{1}\right|
$$

Thus,

$$
\left|\int_{0}^{\rho /\left|\nu_{2}\right|} \int_{0}^{\pi} I(\sigma) d \sigma_{1} d \sigma_{2}\right| \leqq \int_{0}^{\rho /\left|\nu_{2}\right|} \int_{0}^{3 \pi / 2\left|\nu_{1}\right|}|I(\sigma)| d \sigma_{1} d \sigma_{2}
$$

Note that this inequality is also valid if $3 \pi / 2\left|\nu_{1}\right| \geqq \pi$.

By estimating $|I(\sigma)|$ from above with $\exp \left(-k \alpha \sigma^{p}\right)$ in the last double integral, substituting $x\left|\nu_{1}\right|^{-1}$ for $\sigma_{1}$ and $y\left|\nu_{2}\right|^{-1} \rho^{2}$ for $\sigma_{2}$, using (7)(ii) and applying Lemma 1(i) we obtain (27) from (28). Lemma 6 is proved.

Proof of Theorem 2. Let

$$
\begin{aligned}
& h(z)=z_{1}^{2} z_{2}^{2}-\left(\frac{z_{1}-1}{2}\right)^{4}\left(\frac{z_{2}-1}{2}\right)^{4}, \\
& g(w)=w^{5}+\left(\frac{w-1}{2}\right)^{10}, \text { for all complex } w
\end{aligned}
$$

and

$$
f(z)=g\left(z_{1}\right) g\left(z_{2}\right) h(z)
$$

As stated prior to Lemma 4, to prove that $f$ satisfies the inequalities 
given in (ii)-(iv) of Theorem 2 it suffices to show that $f$ satisfies the conditions given in the hypothesis of Theorem 1.

To show that

$$
|f(z)|<1 \text { for } z \neq(1,1), \quad\left|z_{1}\right|=\left|z_{2}\right|=1
$$

it suffices to prove that

$$
|h(z)| \leqq 1 \text { for }\left|z_{1}\right|=\left|z_{2}\right|=1
$$

since by Lemma 4

$$
|g(w)|<1 \text { for } w \neq 1, \quad|w|=1 \text {. }
$$

Since

$$
-\sin ^{2}(\alpha / 2) e^{1 \alpha}=\left(\frac{e^{i \alpha}-1}{2}\right)^{2}
$$

then

$$
h\left(e^{l \sigma}\right)=e^{2 \imath\left(\sigma_{1}+\sigma_{2}\right)}\left(1-\sin ^{4}\left(\sigma_{1} / 2\right) \sin ^{4}\left(\sigma_{2} / 2\right)\right)
$$

from which (29) follows immediately.

To show that $f$ belongs to $\Phi_{4,10}$ an expansion of $f\left(e^{i \sigma_{1}}, e^{i \sigma_{2}}\right)$ about the point $(0,0)$ is needed. By Lemma 4

$$
g\left(e^{i s}\right)=\exp \left(5 s i-\sum_{j=10}^{\infty} d_{s} s^{\jmath}\right), \quad d_{10}>0
$$

for all $s$ in some neighborhood of 0 .

By developing

$$
1-\sin ^{4}\left(\sigma_{1} / 2\right) \sin ^{4}\left(\sigma_{2} / 2\right)
$$

and

$$
\exp \left(-\sum_{\jmath \in Z^{2}} c_{\jmath} \sigma^{\prime}\right)
$$

in power series in $\sigma_{1}$ and $\sigma_{2}$ about $(0,0)$ and comparing these series conclude using (30) that for all $\sigma$ in some neighborhood of $(0,0)$

$$
h\left(e^{\imath \sigma}\right)=\exp \left(2 i\left(\sigma_{1}+\sigma_{2}\right)-\sum_{j, j, 2 \geqq 4} c_{\jmath} \sigma^{\prime}\right), \quad c_{(4,4)}>0,
$$


where the convergence of the double power series is absolute. This, (31) and the definition of $f$ imply that on some neighborhood of $(0,0)$

$$
f=\exp (i \lambda-\psi)
$$

where

$$
\begin{gathered}
\lambda(\sigma)=7\left(\sigma_{1}+\sigma_{2}\right), \\
\psi(\sigma)=\sum_{j_{1}, j_{2} \geqq 4} c_{j} \sigma^{\prime}+\sum_{h \geqq 10} d_{h}\left(\sigma_{1}^{h}+\sigma_{2}^{h}\right), \quad d_{10}, c_{4,4}>0,
\end{gathered}
$$

where again the convergence is absolute.

It follows from Lemma 5 that $\psi$ satisfies (3) with

$$
\phi(\sigma)=c_{(4,4)} \sigma^{4}+d_{10}\left(\sigma_{1}^{10}+\sigma_{2}^{10}\right)
$$

and hence that $f$ belongs to $\Phi_{4,10}$ and satisfies the hypotheses of Theorem 1. This proves Theorem 2(ii)-(iv).

It remains to show that $f$ satisfies the inequality given in Theorem 2(i). For this adopt the notation of Theorem 1 and its proof. Also, let

$$
\begin{aligned}
& S_{l}=\left\{m \in Z^{2}:\left|\nu_{1}\right|<1,\left|\nu_{3-1}\right|>k^{\frac{1}{4}}\right\}, \\
& S_{3}=\left\{m \in Z^{2}: 1 / \log k \leqq \rho^{2} \leqq 1\right\}, \\
& S_{4}=\left\{m \in Z^{2}: \min \left(\rho^{2},\left|\nu_{l}\right|\right) \geqq 1\right\} .
\end{aligned}
$$$$
j=1,2,
$$

It suffices to show for $1 \leqq j \leqq 4$ that

$$
\sum_{S_{1}}\left|\left(f^{k}\right)_{m}\right|=O(\log k), \text { as } k \rightarrow \infty,
$$

since a similar estimate of the sum over the remainder of $Z^{2}$ follows from Theorem 1(ii) and the fact that the cardinality of $Z^{2} \backslash \cup S_{J}$ is $O\left(k^{\frac{1}{4}}\right)$, as $k \rightarrow \infty$.

To prove (35) for $j=1$ twice integrate by parts the integral defining $\left(f^{k}\right)_{m}$, each time taking $\exp \left(i \lambda_{2} \sigma_{2}\right)$ to be the term integrated, and obtain

$$
\left|\left(f^{k}\right)_{m}\right| \leqq \nu_{2}^{-2} k \int|F|^{k-2}\left|(k-1)\left(D_{2} F\right)^{2}+F D_{2}^{2} F\right|,
$$

where

$$
F(\sigma)=f\left(e^{i \sigma}\right) \exp (-i \lambda(\sigma)), \text { for all } \sigma
$$


Choose $\delta>0$ so that (2), (4), (32) and (33) hold on the rectangle $V=(-\delta, \delta)^{2}$ and so that the convergence of the series in (33) is absolute on $V$. Write $I_{\Pi 1}$, the integral in (36), as the sum of integrals over $V$ and $C V$ :

$$
I_{\Pi}=I_{V}+I_{C V}
$$

Since $f$ is a polynomial it follows from (37) and (32) that all partial derivatives of $F$ are continuous and hence bounded on $\Pi$. From (37), (32) and (1) one obtains for some $\gamma$ in $(0,1)$ that $|F| \leqq \gamma$ on $C V$. Thus,

$$
I_{C V}=o\left(\gamma^{k}\right) \text {, as } k \rightarrow \infty \text {. }
$$

From (37) and (2) it follows that $F=\exp (-\psi)$ on $V$ so that the integrand of $\int_{V}$ equals

$$
|F|^{k}\left|k^{2}\left(D_{2} \psi\right)^{2}-k D_{2}^{2} \psi\right|
$$

on $V$. From (37), (32) and (4) one obtains that

$$
|F|=|f| \leqq \exp (-b \phi)
$$

on $V$. Since the convergence of the series in (33) is absolute on $V,(33)$ and (34) imply that

$$
\left|D_{2}^{\prime} \psi\right|<c\left|D_{2}^{\prime} \phi\right|, \quad j=1,2,
$$

on $V$. Using this inequality and (41) to estimate the expression in (40), which is the integrand of $I_{V}$, one obtains

$$
I_{V} \leqq c \int_{V} \exp (-b k \phi)\left(k^{2}\left(D_{2} \phi\right)^{2}+k D_{2}^{2} \phi\right) .
$$

Using (34) to substitute for $\phi$ gives

$$
I_{V} \leqq c \sum \int_{V} k^{\gamma} \sigma^{4 \alpha} \sigma_{2}^{10(\gamma-\alpha)-2} \exp \left(-b k\left(\sigma^{4}+\sigma_{2}^{10}\right)\right),
$$

where the sum is over all $(\gamma, \alpha)$ in $Z^{2}$ such that $\gamma$ belongs to the set $\{1,2\}$ and $0 \leqq \alpha \leqq \gamma$.

Let $S=\left\{\sigma \in V:\left|\sigma_{2}\right|<k^{-\frac{1}{4}} \delta\right\}$ and write $\int_{V}$, given in (42), as

$$
\int_{V}=\int_{S}+\int_{V \backslash S}
$$


Taking supremums over $S$ one obtains that

$$
\left|\int_{S}\right| \leqq c k^{\frac{1}{2}} \int_{S} \exp \left(-k a \sigma^{4}\right)
$$

Substituting $k^{-\frac{1}{4}} u$ for $\sigma_{2}$ in the last integral yields

$$
\left|\int_{S}\right|=O\left(k^{\frac{1}{4}}\right), \text { as } k \rightarrow \infty .
$$

Estimate the integrand of $\int_{V \backslash S}$ by applying the inequality

$$
\exp (-w) \leqq A_{p} w^{-p}, \quad w>0, \quad p>0
$$

first with $w=b k \sigma^{4}, p=\alpha$ and then with $w=b k \sigma_{2}^{10}, p=\gamma-\alpha$ to obtain

$$
\begin{aligned}
\int_{V \backslash S} & \leqq \int_{V \backslash S} \sigma_{2}^{-2} \exp \left(-k b \sigma^{4}\right) \\
& \leqq k^{\frac{1}{2}} \int_{V \backslash S} \exp \left(-k b \sigma^{4}\right)
\end{aligned}
$$

By substituting $u$ for $k^{\frac{1}{4}} \sigma_{2}$ in this last integral and applying Lemma 1(ii) one obtains that

$$
\int_{V \backslash S} \leqq c k^{\frac{1}{4}} \log k
$$

This and (42)-(44) imply that

$$
I_{V} \leqq c \nu_{2}^{-2} k^{\frac{1}{4}} \log k
$$

which with (36), (38) and (39), yields a similar estimate of $\left|\left(f^{k}\right)_{m}\right|$, valid for all $m$ in $S_{1}$.

Since $\left|\nu_{1}\right|<1$ for each $m$ in $S_{1}$ it follows for each $k$ that the set $\left\{m_{1}:\left(m_{1}, m_{2}\right) \in S_{1}\right\}$ has at most two elements. So

$$
\sum_{S_{1}}\left|\left(f^{k}\right)_{m}\right| \leqq c k^{\frac{1}{4}} \log k \sum_{\left|\nu_{2}\right| \geqq k^{1 / 4}} \nu_{2}^{-2}=O(\log k)
$$

as $k \rightarrow \infty$. This proves (35) for $j=1$. The proof for $j=2$ is similar.

Let

$$
N_{i}=\left\{m \in S_{3}: 1 /(i+1)<\rho^{2} \leqq 1 / i\right\}, \quad i=1,2, \cdots
$$


From the fact that the cardinality of $N_{t}$ is less than or equal to $c k^{\frac{1}{4}}(\log k) / i(i+1), i=1,2, \cdots$ and from Lemmas 2 and 6 it follows that

$$
\sum_{N_{i}}\left|\left(f^{k}\right)_{m}\right| \leqq c(\log k)(\log (i+1)+1) / i(i+1), \quad i=1,2, \cdots .
$$

Since $S_{3} \subset \cup_{t<\log k} N_{t}$, this last inequality implies that

$$
\begin{array}{r}
\sum_{S_{3}}\left|\left(f^{k}\right)_{m}\right| \leqq c \log ^{2} k \sum_{i \leqq \log k}(\log (i+1)+1) / i(i+1) \\
=O(\log k), \text { as } k \rightarrow \infty
\end{array}
$$

This proves (35) for $j=3$.

To prove (35) for $j=4$ it suffices to prove that

$$
\left|\left(f^{k}\right)_{m}\right| \leqq c k^{\frac{1}{4}}\left(\nu_{1} \nu_{2}\right)^{-2} \text { for all } m \text { in } S_{4}
$$

since the sum of $\left(\nu_{1} \nu_{2}\right)^{-2}$ over the set $\left\{m \in S_{4}:\left|\nu_{t}\right| \geqq k\right\}$ is $O\left(k^{-2}\right)$, as $k \rightarrow \infty$, and over its complement in $S_{4}$ is $O\left(k^{-\frac{1}{4}} \log k\right)$, as $k \rightarrow \infty$. For the last estimate one uses that $1 \leqq\left|\nu_{\imath}\right| ; 1 \leqq \rho=\left|\nu_{1} \nu_{2}\right| k^{-\frac{1}{4}}$, for all $m$ in $S_{4}$.

To prove (46) integrate by parts the integral defining $\left(f^{k}\right)_{m}$, twice each with respect to $\sigma_{1}$ and $\sigma_{2}$, each time taking $e^{i \nu \cdot \sigma}$ to be the term integrated and obtain

$$
\left(f^{k}\right)_{m}=\left(2 \pi \nu_{1} \nu_{2}\right)^{-2} \int e^{i \nu \cdot \sigma} \mathscr{D} F^{k} d \sigma
$$

where $F$ is defined by (37) and $\mathscr{D}$ is the differential operator $D_{2}^{2} D_{1}^{2}$. Since $m$ belongs to $S_{4}, \nu_{1} \nu_{2} \neq 0$.

Let $H=F \cdot \exp (-\phi)$ on $R^{2}$. Then

$$
\left(f^{k}\right)_{m}\left(2 \pi \nu_{1} \nu_{2}\right)^{2} \leqq \sum_{i=1}^{4} J_{i}
$$

where

$$
\begin{aligned}
& J_{1}=\int_{C V}\left|\mathscr{D} F^{k}\right|, \\
& J_{2}=\int_{V}\left|\mathscr{D} F^{k}-H^{k} \mathscr{D} \exp (-k \phi)\right|, \\
& J_{3}=\int_{V}\left|\left(H^{k}-1\right) \mathscr{D} \exp (-k \phi)\right|, \\
& J_{4}=\left|\int_{V} e^{i v \cdot \sigma} \mathscr{D} \exp (-k \phi)\right| .
\end{aligned}
$$


Since $F$ and its derivatives are continuous and since $|F| \leqq 1$ on $\Pi$ with equality only at the point $(0,0)$ there exists some $\delta$ in $(0,1)$ such that

$$
\sup _{m} J_{1}=O\left(\delta^{k}\right) \text {, as } k \rightarrow \infty \text {. }
$$

To estimate $J_{2}$ notice that on $V$

$$
\mathscr{D} F^{k}-H^{k} \exp (-k \phi)=\left(\sum_{\jmath=1}^{4} k^{\prime} s_{j}\right) F^{k}
$$

where for $1 \leqq j \leqq 4 s_{J}$ is a double power series in $\sigma_{1}$ and $\sigma_{2}$, absolutely convergent on $V$, with no terms of degree less than $8 j-3$. Using (41) and (34) to estimate $F$ on $V$ yields

$$
\left|J_{2}\right| \leqq \sum_{j=1}^{4} k^{\prime} \int_{V} s_{\jmath} \exp \left(-b k \sigma^{4}\right) d \sigma
$$

Substituting $\tau k^{-1 / 8}$ for $\sigma$ yields

$$
\left|J_{2}\right| \leqq c k^{1 / 8} \int_{k^{1 / 8} V} \exp \left(-b \tau^{4}\right) d \tau
$$

since $s_{\jmath}$ is continuous on $V$ with terms of degree at least $8 j-3$. So by Lemma 1(ii),

$$
\sup _{m}\left|J_{2}\right|=O\left(k^{1 / 8} \log k\right) \text {, as } k \rightarrow \infty \text {. }
$$

To estimate $J_{3}$ let $W_{k}$ be defined as in (10), with $c=\frac{1}{4}$, and write

$$
J_{3}=\left(\int_{W_{k}}+\int_{V \backslash W_{k}}\right)\left|\left(H^{k}-1\right) \mathscr{D} \exp (-k \phi)\right|
$$

By (14),

$$
\sup _{W_{k}}\left|H^{k}-1\right|=O\left((\log k)^{-1}\right) \text {, as } k \rightarrow \infty \text {. }
$$

So

$$
\int_{W_{k}} \leqq c(\log k)^{-1} \int_{\Pi} \mathscr{D} \exp (-k \phi)
$$


To estimate this last integral notice that

$$
\mathscr{D} \exp (-k \phi)=\sum_{j=1}^{4} k^{\prime} p_{\jmath}(\sigma) \exp (-k \phi)
$$

where $p_{\text {, }}$ is a polynomial in two variables, each term of which is of degree at least $8 j-4$. By replacing $\mathscr{D} \exp (-k \phi)$ in the last integral with the equivalent expression given in (51) and then by substituting $\tau k^{-1 / 8}$ for $\sigma$ one obtains that

$$
\int_{W_{k}}<c(\log k)^{-1} k^{\frac{1}{4}} \int_{k^{1 / 8} \Pi} \exp \left(-k \tau^{4}\right) d \tau
$$

Applying Lemma 1(ii) then yields

$$
\int_{W_{k}}=O\left(k^{\frac{1}{4}}\right), \text { as } k \rightarrow \infty .
$$

To estimate $\int_{V \backslash W_{k}}$, as given in (50), note that $|H| \leqq 1$ since $H=$ $F \cdot \exp (-\phi)$ and hence that

$$
\int_{V \backslash W_{k}} \leqq 2 \int_{V \backslash W_{k}}|\mathscr{D} \exp (-k \phi)|
$$

From (51) obtain that on $V$

$$
|\mathscr{D} \exp (-k \phi)| \leqq a k^{4} \exp (-k \phi)
$$

But $W_{k}$ is defined by (10) with $c=\frac{1}{4}$ so

$$
\sup _{V \backslash W_{k}} \exp (-k \phi) \leqq a k^{-5}
$$

From these last two inequalities and (51) conclude that

$$
\int_{V \backslash W_{k}}=O\left(k^{-1}\right), \text { as } k \rightarrow \infty
$$

which with (52) and (50) implies that

$$
\sup _{m} J_{3}=O\left(k^{\frac{1}{4}}\right) \text {, as } k \rightarrow \infty \text {. }
$$


From this estimate and (47)-(49) conclude that to prove (46) and hence Theorem 2 it suffices to show that

$$
\sup _{m \in J_{4}} J_{4}=O\left(k^{\frac{1}{4}}\right) \text {, as } k \rightarrow \infty \text {. }
$$

For this write

$$
J_{4}=\left|\left(-\int_{S}+\int_{D}+\sum_{i=1}^{2} \int_{E_{i} \cup S}\right) \chi_{v} e^{i v \cdot \sigma} \mathscr{D} \exp (-k \phi)\right|,
$$

where $S, D, E_{\iota}, i=1,2$ are defined in the proof of Lemma 2 .

Using the identity in (51) to substitute for $\mathscr{D} \exp (-k \phi)$ in $\int_{S}$ and then substituting $\tau k^{-1 / 8}$ for $\sigma$ obtain

$$
\left|\int_{S}\right| \leqq c k^{\frac{1}{4}} \int_{k^{1 / 8} S} \exp \left(-b \tau^{4}\right)
$$

Since the area of $S$ equals $4 \rho^{2}\left|\nu_{1} \nu_{2}\right|^{-1}$, which by 7 (ii) is $O\left(k^{-\frac{1}{4}}\right)$, as $k \rightarrow \infty$, we deduce from the last inequality that

$$
\left|\int_{S}\right|=O\left(k^{\frac{1}{4}}\right), \text { as } k \rightarrow \infty \text {. }
$$

In the same way that inequality (56) was derived obtain

$$
\left|\int_{D}\right| \leqq c k^{\frac{1}{4}} \int_{k^{1 / 8} D} \exp \left(-b \tau^{4}\right) .
$$

Since $\exp \left(-b \tau^{4}\right)$ is integrable on $(1, \infty)^{2}$ conclude that

$$
\left|\int_{D}\right|=O\left(k^{\frac{1}{4}}\right), \text { as } k \rightarrow \infty .
$$

From this estimate, (57) and (55) conclude that to prove (54) and hence Theorem 2 it suffices to show that

$$
\sup _{m \in S_{4}}\left|\int_{E_{1} \cup S} \chi_{V} \cdot e^{i v \sigma} \mathscr{D} \exp (-k \phi)\right|=O\left(k^{\frac{1}{4}}\right) \text {, as } k \rightarrow \infty,
$$

for $i=1,2$. The proof of (58) is given for $i=1$, the proof for $i=2$ being similar.

Since $\chi_{V} \cdot \mathscr{D} \exp (-k \phi)$ is an even function of $\sigma_{1}$ and of $\sigma_{2}$ the integral in (58) equals 


$$
4 \int_{0}^{\rho /\left|\nu_{2}\right|} \int_{0}^{\infty} \chi_{V} \cdot \cos \left(\nu_{1} \sigma_{1}\right) \cos \left(\nu_{2} \sigma_{2}\right) \mathscr{D} \exp (-k \phi) d \sigma_{1} d \sigma_{2}
$$

Using (34) to express $\phi$ in terms of $\sigma$ and then calculating $\mathscr{D} \exp (-k \phi)$ conclude that for some $\delta$ in $(0,1)$

$$
\int_{C V}|\mathscr{D} \exp (-k \phi)|=O\left(\delta^{k}\right), \text { as } k \rightarrow \infty,
$$

and that $\mathscr{D} \exp (-k \phi)$ can be expressed as a finite linear combination of terms of the form

$$
k^{L+P+Q}\left(\sigma_{1}^{L} \sigma_{2}^{P}\right)^{10} \sigma^{4 Q-2} \exp (-k \phi),
$$

where $P, Q, L$ are nonnegative integers satisfying $10 \min (L, P)+4 Q-$ $2 \geqq 0$. Therefore, letting $E(\sigma)=\sigma_{1}^{10 L+4 O-2} \exp (-k \phi)$,

$$
I_{n}\left(\sigma_{2}\right)=\int_{0}^{n \pi / 2\left|\nu_{1}\right|} \cos \left(\nu_{1} \sigma_{1}\right) E(\sigma) d \sigma_{1}
$$

for all positive integers $n$, and $I_{\infty}\left(\sigma_{2}\right)=\lim _{n \rightarrow \infty} I_{n}\left(\sigma_{2}\right)$ conclude that to prove $(58)$ it suffices to show that

$$
\sup _{m \in S_{4}} k^{L+P+Q}\left|\int_{0}^{\rho /\left|\nu_{2}\right|} \cos \left(\nu_{2} \sigma_{2}\right) \sigma_{2}^{10 P+4 Q-2} I_{\infty}\left(\sigma_{2}\right) d \sigma_{2}\right|=O\left(k^{\frac{1}{4}}\right),
$$

as $k \rightarrow \infty$, since the integral in (59), which equals the integral in (58), can be written as a finite linear combination of integrals of the type given in (60) plus a term that is $O\left(\delta^{k}\right)$, as $k \rightarrow \infty$, where $0<\delta<1$.

To prove (60) first fix $\sigma_{2}$ in $\left(0, \rho /\left|\nu_{2}\right|\right)$, consider $E$ to be a function of the one variable $\sigma_{1}$ and estimate $I_{\infty}\left(\sigma_{2}\right)$. E has at most one critical point on $(0, \infty)$ since $\phi$ is as in (34) and since $10 L+4 Q-2 \geqq 0$. In any case there exists a smallest nonnegative integer $r$ such that the function $E$ is monotone decreasing on $\left(0, \pi(4 r+1) / 2\left|\nu_{1}\right|\right)$.

Since $E$ is monotone decreasing on $\left(\pi(4 r+1) / 2\left|\nu_{1}\right|, \infty\right)$ it follows that $I_{\infty}-I_{4 r+1}$ and $I_{\infty}-I_{4 r+3}$ are of opposite signs so that

$$
\left|I_{\infty}-I_{4 r+1}\right| \leqq\left|I_{4 r+1}-I_{4 r+3}\right| \leqq \pi S /\left|\nu_{1}\right|
$$

where

$$
S=\sup _{\sigma_{1} \in(0, \infty)}|E| .
$$


If $r<3$, then $\left|I_{4 r+1}\right| \leqq 9 \pi S / 2\left|\nu_{1}\right|$.

If $r \geqq 3$, then $E$ is monotone increasing on $\left(0, \pi(4 r-5) / 2\left|\nu_{1}\right|\right)$ so that $I_{4 r-7}-I_{1}$ and $I_{4 r-5}-I_{3}$ are of opposite signs. Thus,

$$
\left|I_{4 r-7}-I_{1}\right| \leqq\left|I_{4 r-5}-I_{4 r-7}+I_{1}-I_{3}\right| \text {. }
$$

So, in the case $r \geqq 3$,

$$
\begin{aligned}
\left|I_{4 r+1}\right| & \leqq\left|I_{4 r+1}-I_{4 r-7}\right|+\left|I_{4 r-5}-I_{4 r-7}\right|+\left|I_{3}-I_{1}\right|+\left|I_{1}\right| \\
& \leqq 13 \pi S / 2\left|\nu_{1}\right|
\end{aligned}
$$

where $S$ is defined by (62). These two estimates of $\left|I_{4 r+1}\right|$ and the inequality (61) imply that $\left|I_{\infty}\right| \leqq 15 \pi S / 2\left|\nu_{1}\right|$. Thus, to prove (60) it suffices to show that

$$
k^{L+P+Q} \sup _{m \in S_{4}}\left(\rho /\left|\nu_{1} \nu_{2}\right|\right) \sup _{R^{2}}\left(\sigma_{2}^{10 P+4 Q-2} E(\sigma)\right)=O\left(k^{\frac{1}{4}}\right),
$$

as $k \rightarrow \infty$.

For all $m$ in $S_{4}, \rho \geqq 1$ which with (7) implies that

$$
\sup _{m \in S_{4}} \rho /\left|\nu_{1} \nu_{2}\right|=\rho^{-1} k^{-\frac{1}{4}} \leqq k^{-\frac{1}{4}}
$$

To estimate the second supremum in (63) and thereby prove (63) in the case $L \geqq P$ use (34) to express $\exp (-k \phi)$ in terms of $\sigma$ and apply inequality (45) first with

$$
w=k \sigma^{4}, \quad p=(10 P+4 Q-2) / 4
$$

and then with

$$
w=k \sigma_{1}^{10}, \quad p=L-P
$$

to obtain that the second supremum in (63) is less than or equal to $c k^{\frac{1}{2}-P / 2}$. This fact with (64) proves (63) in the case $L \geqq P$. By interchanging the roles of $L$ and $P$ in estimating the second supremum in (63) one obtains a proof of $(63)$ in the case $P \geqq L$.

The author wishes to thank his doctoral advisor, Professor Bogdan Baishanski, for his guidance and the referee for his helpful suggestions. 


\section{REFERENCES}

1. B. Bajsanski, Sur une classe générale de procédés de sommations du type d'Euler-Borel, Acad. Serbe Sci. Publ. Inst. Math., 10 (1956), 131-152.

2. J. Clunie and P. Vermes, Regular Sonnenschein type summability methods, Acad. Roy. Belg. Bull. Cl. Sci., 45 (1959), 930-954.

3. D. Girard, The asymptotic behavior of norms of powers of absolutely convergent Fourier series, Pacific J. Math., 37 (1971), 357-381.

4. G. W. Hedstrom, Norms of Powers of Absolutely Convergent Fourier Series in Several Variables, Mich. Math. J., 14 (1967), 493-495.

5. G. W. Hedstrom, Norms of powers of Fourier transforms, Michigan Math. J., 13 (1966), 249-259.

6. C. Heiberg, Norms of powers of absolutely convergent Fourier series of several variables, J. of Functional Analysis, 14 (1973), 382-400.

7. D. J. Newman, Homomorphisms of 1+, Amer. J. Math., 91 (1969), 37-46.

8. B. M. Schreiber, Measures with bounded convolution powers, Trans. Amer. Math. Soc., 151 (1970), 405-431.

9. W. G. Strang, Polynomial approximation of Bernstein type. Trans. Amer. Math. Soc., 105 (1962), 525-535.

10. V. Thomee, Stability of difference schemes in the maximum-norm, J. Differential Equations, 1 (1965), 273-292.

Received February 14, 1975. This paper is based on a portion of the author's doctoral dissertation, which was written at The Ohio State University and partially supported by N.S.F. Grant \# G.P. 8959.

Virginia Wesleyan College-Norfolk, VA. 



\section{Pacific Journal of Mathematics}

\section{Vol. 66, No. $1 \quad$ November, 1976}

Helen Elizabeth. Adams, Factorization-prime ideals in integral domains ............ Patrick Robert Ahern and Robert Bruce Schneider, The boundary behavior of Henkin's kernel.

Daniel D. Anderson, Jacob R. Matijevic and Warren Douglas Nichols, The Krull

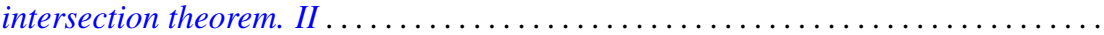

Efraim Pacillas Armendariz, On semiprime P.I.-algebras over commutative regular

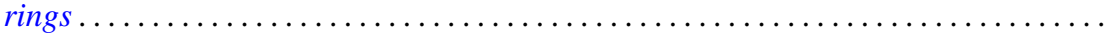

Robert H. Bird and Charles John Parry, Integral bases for bicyclic biquadratic fields

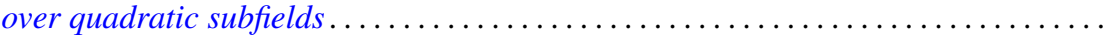

Tae Ho Choe and Young Hee Hong, Extensions of completely regular ordered

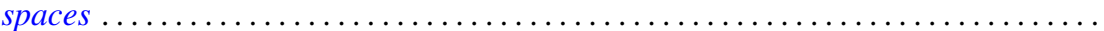

John Dauns, Generalized monoform and quasi injective modules ...............

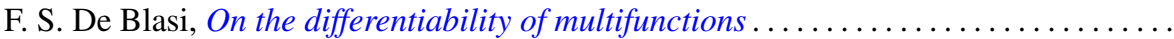

Paul M. Eakin, Jr. and Avinash Madhav Sathaye, R-endomorphisms of $R[[X]]$ are

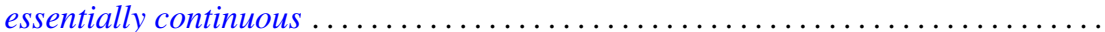

Larry Quin Eifler, Open mapping theorems for probability measures on metric

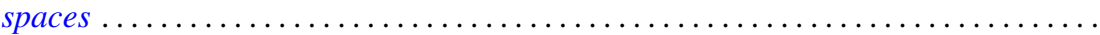

Garret J. Etgen and James Pawlowski, Oscillation criteria for second order self adjoint

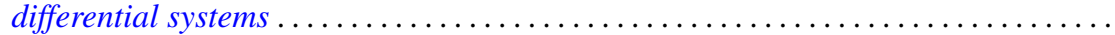

Ronald Fintushel, Local $S^{1}$ actions on 3-manifolds .

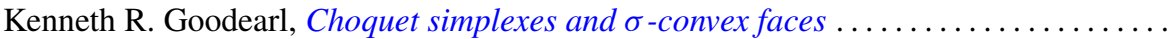

John R. Graef, Some nonoscillation criteria for higher order nonlinear differential

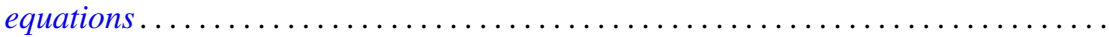

Charles Henry Heiberg, Norms of powers of absolutely convergent Fourier series: an example.

Les Andrew Karlovitz, Existence of fixed points of nonexpansive mappings in a space

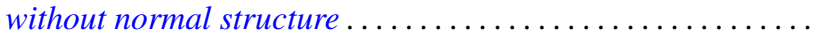

Gangaram S. Ladde, Systems of functional differential inequalities and functional

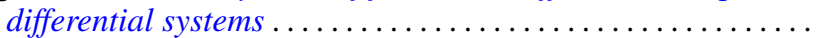

Joseph Michael Lambert, Conditions for simultaneous approximation and interpolation with norm preservation in $C[a, b]$.

Ernest Paul Lane, Insertion of a continuous function.

Robert F. Lax, Weierstrass points of products of Riemann surfaces .

Dan McCord, An estimate of the Nielsen number and an example concerning the Lefschetz fixed point theorem...

Paul Milnes and John Sydney Pym, Counterexample in the theory of continuous functions on topological groups...

Peter Johanna I. M. De Paepe, Homomorphism spaces of algebras of holomorphic functions

Judith Ann Palagallo, A representation of additive functionals on $L^{p}$-spaces,

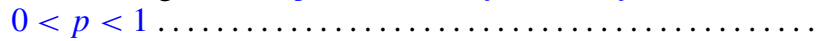

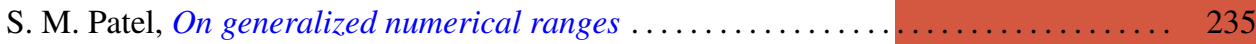

Thomas Thornton Read, A limit-point criterion for expressions with oscillatory

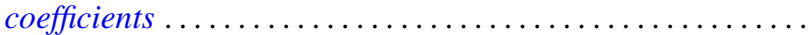

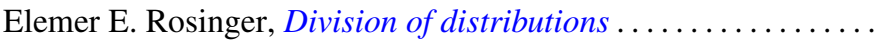

Peter S. Shoenfeld, Highly proximal and generalized almost finite

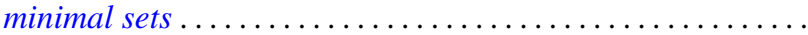

R. Sirois-Dumais and Stephen Willard, Quotient-universal sequential spaces

Robert Charles Thompson, Convex and concave functions of singular values of matrix sums....

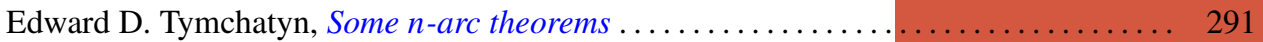

